\title{
A kinematic, flexure-based mechanism for precise, parallel motion for the Hertz Variable-delay Polarization Modulator (VPM)
}

\author{
G. M. Voellmer ${ }^{*}{ }^{a}$, D.T. Chuss ${ }^{\mathrm{a}}$, M. Jackson ${ }^{\mathrm{a}}$, M. Krejny ${ }^{\mathrm{b}}$,S.H. Moseley ${ }^{\mathrm{a}}$, G. Novak ${ }^{\mathrm{b}}$, E.J. \\ Wollack ${ }^{\mathrm{a}}$, $\mathrm{NASA}$ GSFC, Greenbelt, MD 20771 \\ ${ }^{b}$ Dept. of Physics and Astronomy, Northwestern University, 2131 Tech Drive \\ Evanston, IL 60208
}

\begin{abstract}
We describe the design of the linear motion stage for a Variable-delay Polarization Modulator (VPM) and of a grid flattener that has been built and integrated into the Hertz ground-based, submillimeter polarimeter. VPMs allow the modulation of a polarized source by controlling the phase difference between two linear, orthogonal polarizations. The size of the gap between a mirror and a very flat polarizing grid determines the amount of the phase difference. This gap must be parallel to better than $1 \%$ of the wavelength. A novel; kinematic, flexurebased mechanism is described that passively maintains the parallelism of the mirror and the grid to $1.5 \mu \mathrm{m}$ over a $150 \mathrm{~mm}$ diameter, with a $400 \mu \mathrm{m}$ throw. A single piezoceramic actuator is used to modulate the gap, and a capacitive sensor provides position feedback for closed-loop control. A simple device that ensures the planarity. of the polarizing grid is also described. Engineering results from the deployment of this device in the Hertz instrument April 2006 at the Submillimeter Telescope Observatory (SMTO) in Arizona are presented.
\end{abstract}

\section{INTRODUCTION}

The polarization of cosmic, submillimeter $(350 \mu \mathrm{m}-850 \mu \mathrm{m})$ radiation is used to study gravity wave traces in: the cosmic microwave background (reference 1) and the magnetic fields in interstellar dust clouds (reference 2) Typically, the signals are very faint compared to the atmospherric background, and the polarized component of those signals is small. To get a useful signal, the polarized component must be modulated at a known frequency with respect to the background. The data system locks on to this frequency and rejects all others; : and a measurement of the polarized component can be obtained.

We have developed a novel method of modulating the polarization (reference 3 ): a polarizing filter made of closely spaced, metal wires, called a "grid", is placed in front of a mirror, with a precise, parallel spacing between them. The grid reflects the component of the incoming radiation that is polarized along the wire's length, and transmits the perpendicular component. The latter is reflected off the mirror; and recombines with the first component, albeit with a longer path. If the delay is half a wavelength, the recombined radiation's effective polarization direction is flipped along the axis of the wire. If the delay is a full wavelength, the polarization direction is unchanged. This drives the requirement on the throw of the modulator linear motion stage, to go from a null \#\#400um\#\# Variable-delay Polarization Modulator (VPM).

During an observation, the VPM (figures 1) is placed in a collimated portion of the beam and the mirror is translated back and forth, varying the delay between the half -wavelength and the full-wavelength conditions at a known frequency. Inside the Hertz instrument, an analyzer grid in front of the detector preferentially blocks one of the ensuing polarization states. In this fashion, the polarized component of the beam is modulated.

*voellmer@gsfc.nasa.gov; phone 301-286-8182 


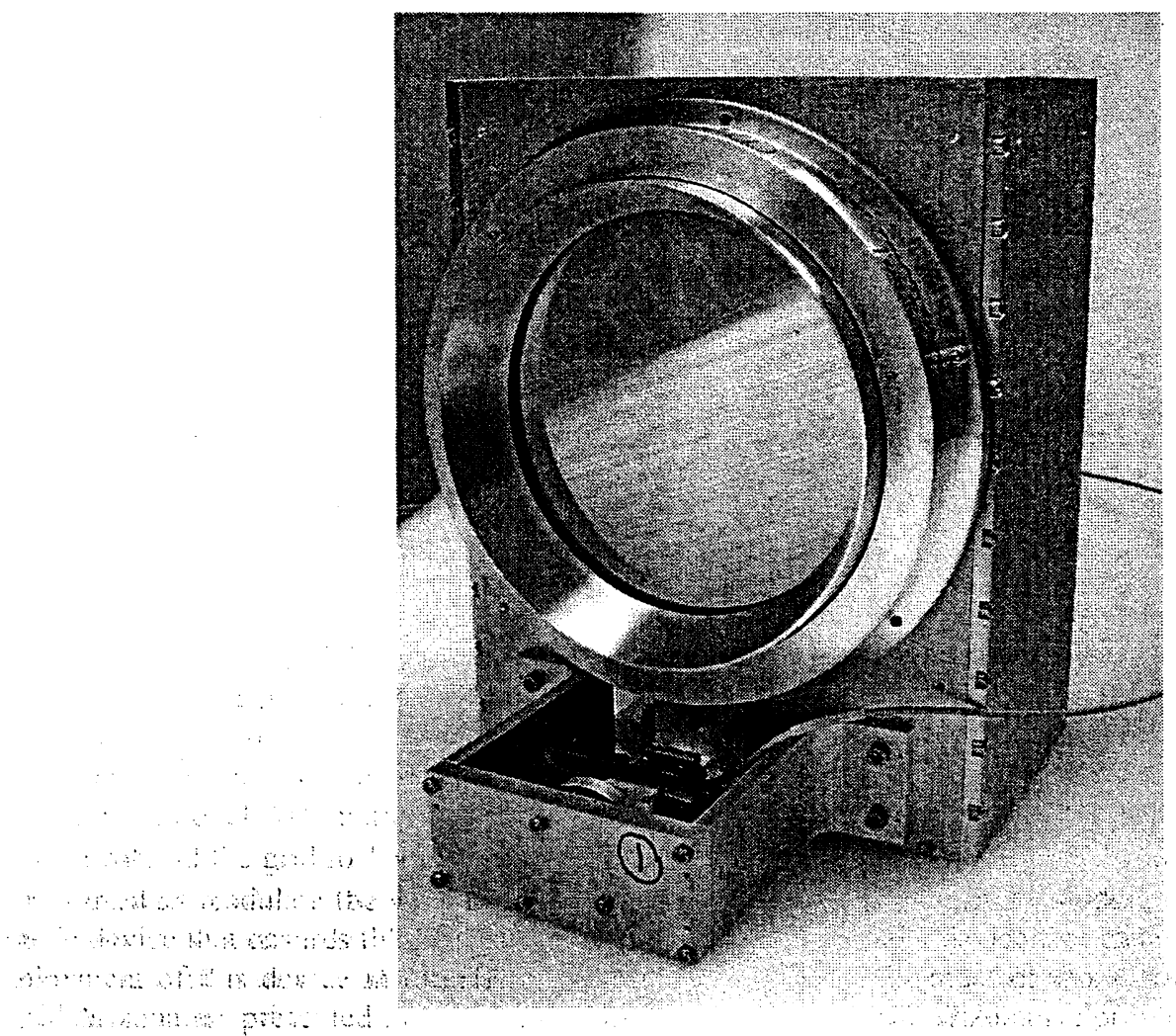

Figure 1): The Variable-delay Polarization Modulator

\section{MODULATOR DESIGN}

In order for the modulator to work efficiently, the spacing between the mirror and the grid must be constant to within $1 \%=2 \%$ of the wavelength of the incoming light over the entire area of the beam. The motion of the gap must be highly linear, with no tilting, and the grid and mirror must be very flat.

The principal components of the Hertz variable-delay polarization modulator are shown in figure 2 . The design is based on the SPIFI Fabry-Perot interferometer (reference 4). The forward optical element, in our case the grid assembly (see also figure 7 ), is magnetically attracted to a rectangular frame structure, while three fine tip / tilt adjustment set screws push it away, thereby setting the coarse gap and the tip and tilt The rear optical element, in our case the mirror; is attached to a moving stage, which is mounted to the main structure via a double blade flexure linear bearing. A linear piezoceramic actuator varies the gap between the two elements; with the position feedback for closed-loop control provided by a capacitive sensor. 


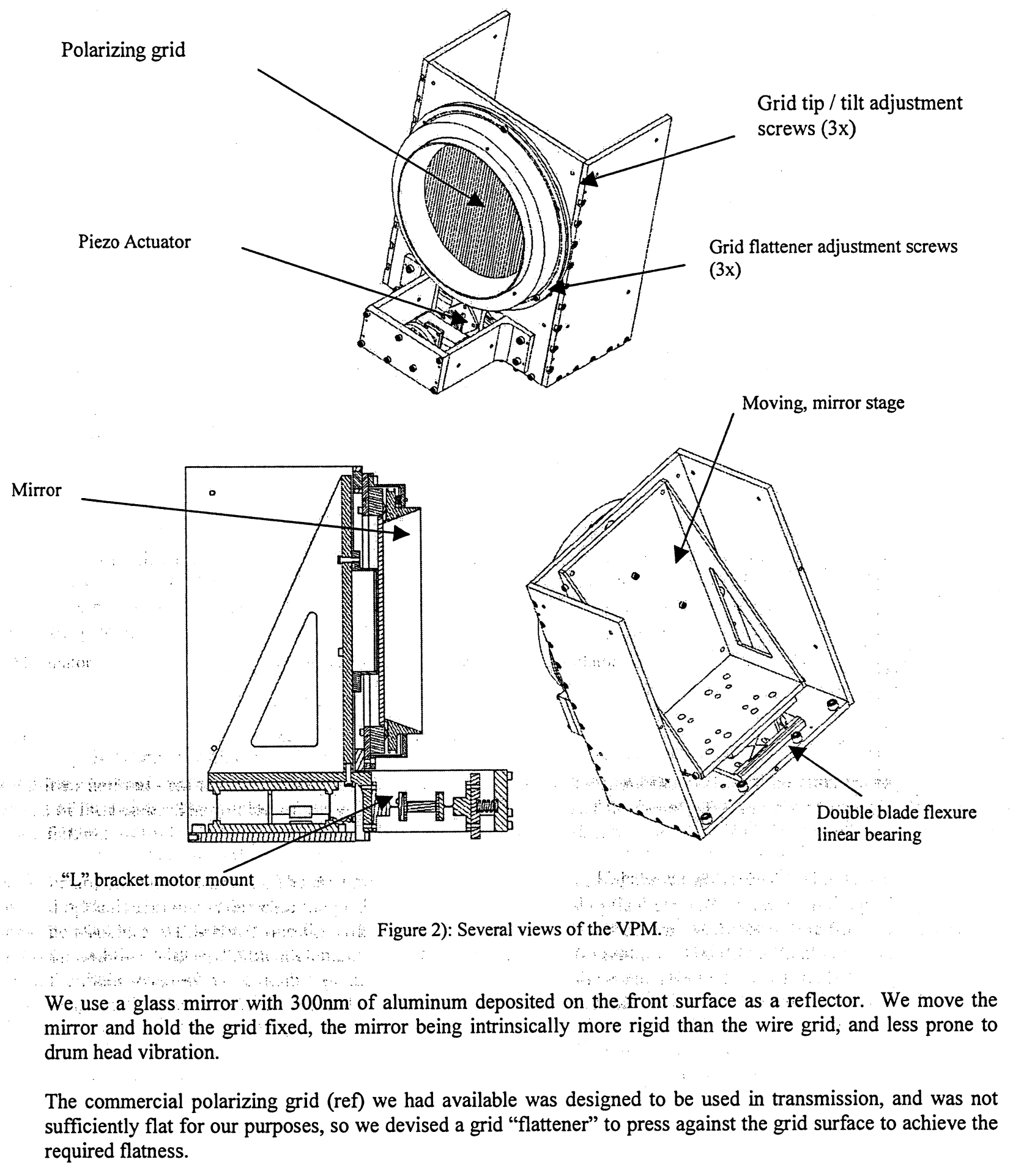




\section{LINEAR FLEXURE STAGE}

The mirror is attached to the body of the modulator via a unique, kinematic double blade flexure, which allows it to move only in the required direction. Although the Hertz VPM did not operate cryogenically, this linear stage was designed and built to do so as a test for possible use in a cryogenic Fabry-Perot interferometer.

Other parallel plate interferometric devices (refxxx) use three linear actuators equally spaced around the optical elements to establish plate spacing, tip and tilt. These have fairly complex control systems, as there is crosscoupling between the controlled axes. This has lead to position noise and lower control bandwidth. For this reason, we decided to try maintaining the parallelism passively, using a double blade flexure, and use a single actuator to set the gap.

A traditional double-blade flexure has two wide blades connected at their ends by rigid plates (figure 3 ). They are usually able to maintain fairly accurate parallelism between the two plates. The width of the blades poses a problem, however, when very precise motion is required, especially when there will be significant temperature changes, such as in cryogenic applications. The width of the first blade locates the top and bottom plates in rotation about the axis perpendicular to the face of the blade, and the second blade overconstrains it. Hence, a double blade flexure is not kinematic.

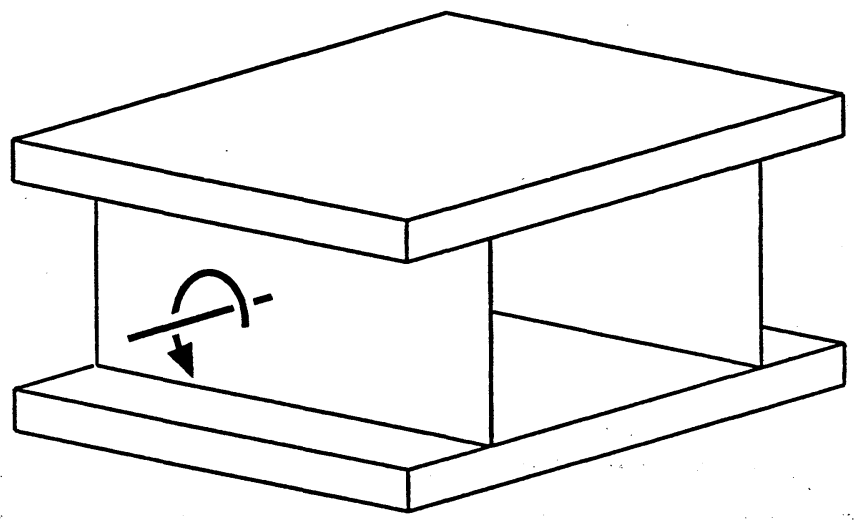

Figure 3): The two blades of a traditional double-blade flexure overconstrain the two plates in rotation.

By machining the blades in an hourglass form, as shown in figure 4, the blades do not constrain the top plate in rotation. To keep the flexure bearing from tipping to the side, a single "outrigger" one-degree-of-constraint flexure is provided. 


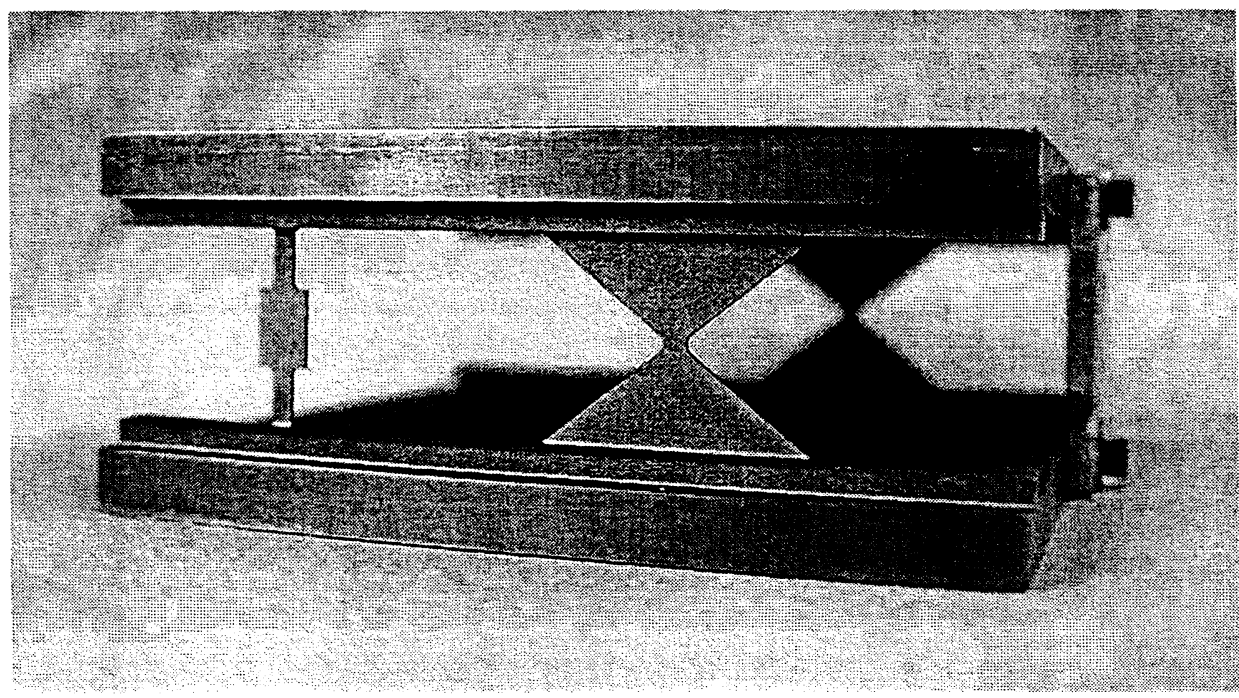

Figure 4): A novel, kinematic double-blade flexure. Hourglass-shaped blades prevent rotation overconstraint, and the outrigger post uniquely constrains it.

An additional problem with traditional double blade flexures is that internal stresses in the blade itself generally cause it to be slightly curved or cupped. As the flexure is deflected, particularly through its neutral point, this cupping "pops through", usually not in a repeatable fashion, and causes positioning errors. By machining the flexure blades in an hourglass form, the area of the blade, and thereby its opportunity to exhibit cupping, is greatly reduced.

The entire flexure assembly shown in figure 4 was machined out of a single billet of Titanium using wire Electrical Discharge Machining (EDM). Cutting the device out of a single billet ensured that there were no thermal expansion coefficient mismatches between parts. The flexure was designed such that it could be manufactured entirely using wire EDM with two setups 90 degrees apart. The outline and interior spaces were rough cut to $1 / 8$ " of the final dimensions before annealing the piece. The final wire EDM cut then had negligible internal stresses. A mechanical hard stop is fitted between the moving plates of the flexure at the machine shop, to prevent the flexure from overtravel overstresses during handling, assembly and testing.

By making the flexure assembly out of one piece of stress-relieved titanium, and by designing the flexure system to be kinematic, we hope to be able to align the optical elements warm, and dispense with the tip / tilt actuators required for a cold alignment peak-up that other designs require.

A commercial piezoceramic actuator and control system (reference 5) provide the required $400 \mu \mathrm{m}$ throw and $<1 \mu \mathrm{m}$ accuracy. A capacitive sensor (reference 6) (figure 5) closes the position loop. The actuator is attached to the moving stage via an " $L$ " bracket such that its line of action passes through the midpoint of the flexure blades (see figure 2). It can be shown that this configuration produces the minimum tipping of the flexure stage as the translational force is applied. The linear actuator resembles a scissors jack (figure 6), and the two ends are mounted via crossed flexure universal joints, to prevent any non-parallel motion of the actuator mounting ends from affecting the parallelism of the mirror stage. 


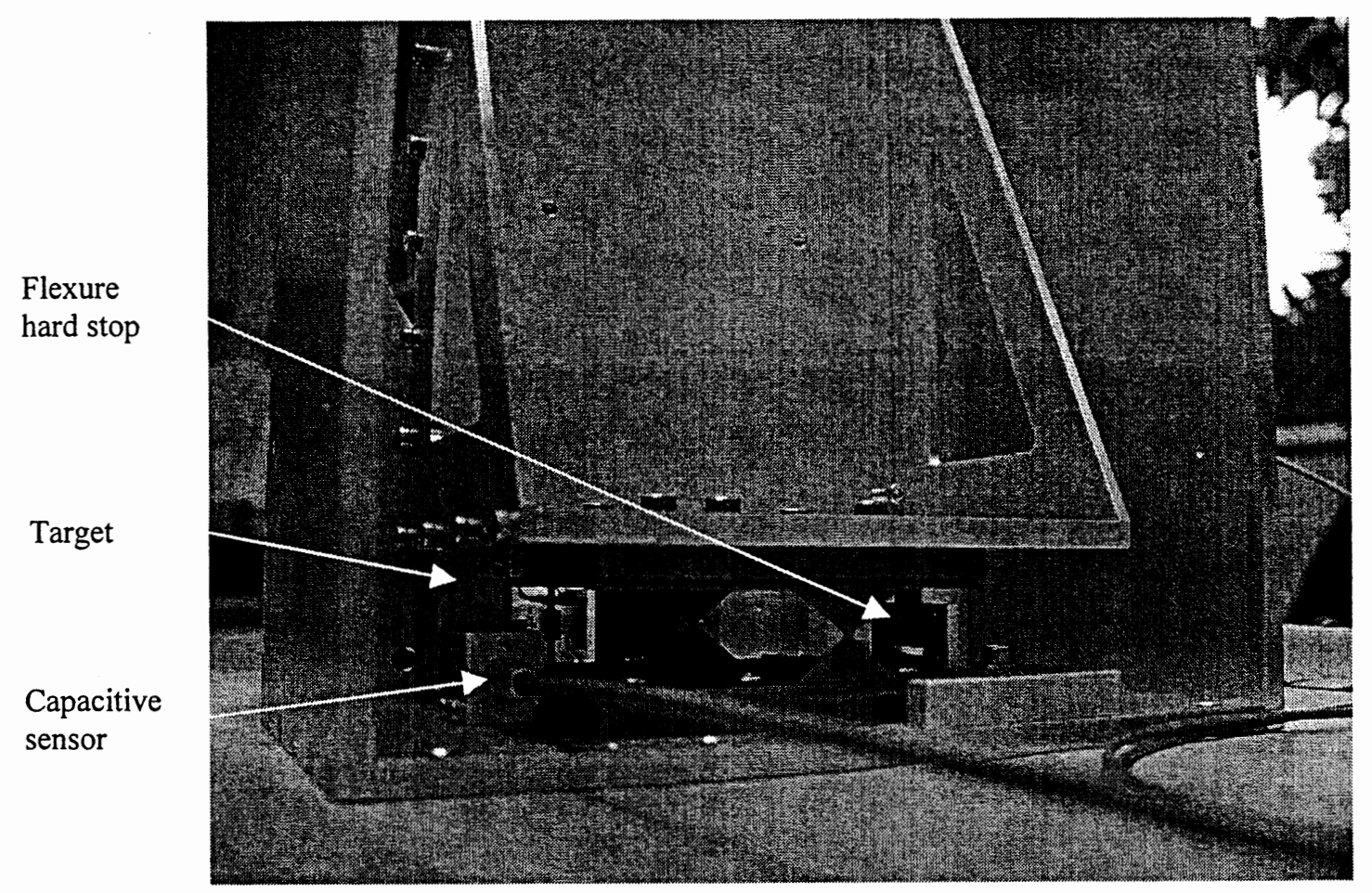

Figure 5): The flexure in place between the mirror stage and the main frame. The capacitive sensor is clamped to the base of the flexure, and the target is mounted to the moving part.

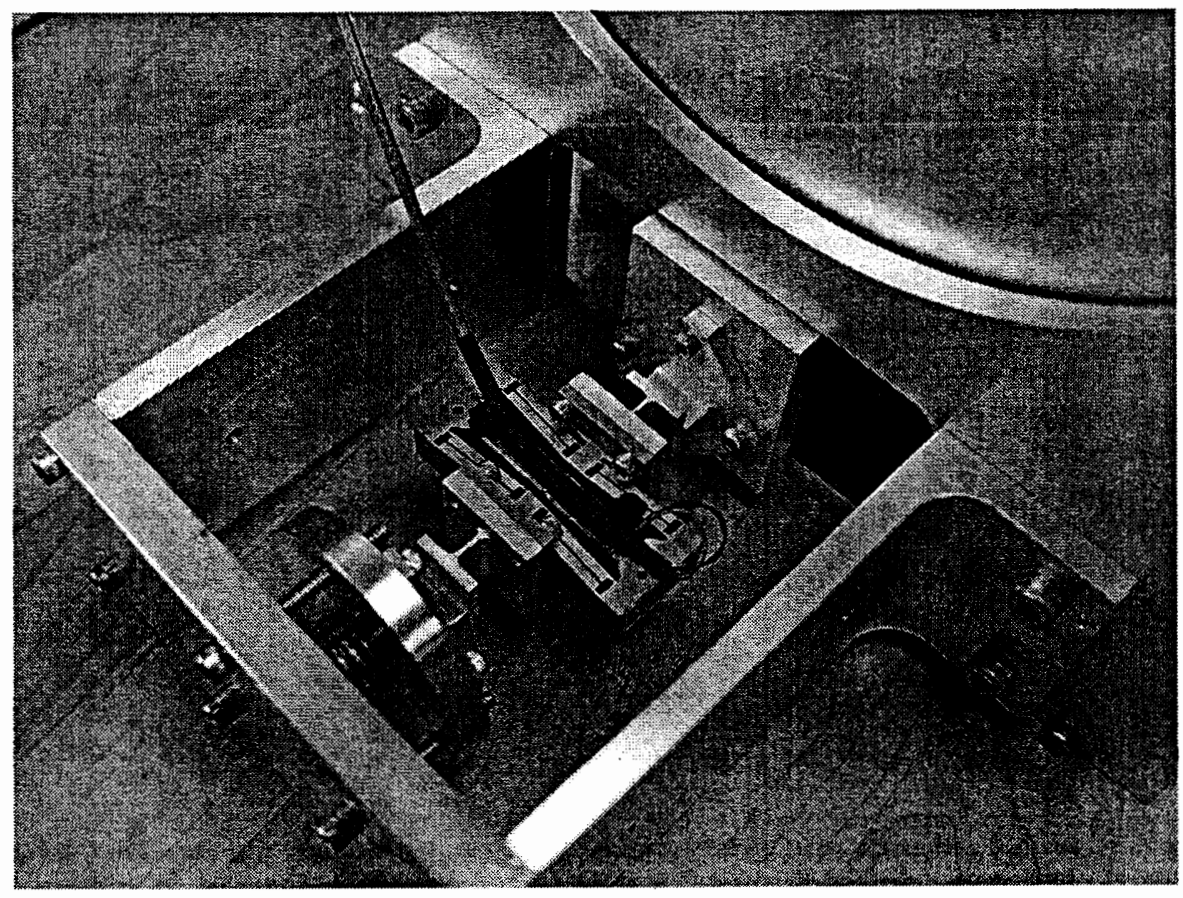

Figure 6): The piezoceramic actuator mounted via crossed flexure universal joints to an adjustment base, on the left, and the " $\mathrm{L}$ " bracket, on the right. 
The linear stage was designed to deflect $500 \mu \mathrm{m}$ from its neutral position in either direction. In use, the stage is only used in one direction, and it is never allowed to go through its neutral position, further minimizing travel through any nonlinear, cupped states.

\section{GRID FLATTENER}

Polarizing grids for the $350 \mu \mathrm{m}$ wavelength that Hertz works in consist of a series of parallel, conductive wires .001 " in diameter, set on .003 " centers. The parallelism and planarity of these wires is critical to the polarization efficiency of the grid. Grids are manufactured by stretching wires over a stretching frame, with spacer teeth to ensure even spacing. Once all the wires are stretched, a ring is glued or soldered to the stretched wire surface, and the excess wires are cut away. The stretching frame can then be reused. To keep the wires straight and parallel, they are under significant tension, generally $50 \%$ of their breaking strength. This imparts a substantial, non-uniform stress to the grid ring, which can cause it to "potato chip" out of planarity. For grids used in transmission, this is not a concern, but in reflection, this would impart an unacceptable phase error to the beam. Our solution was to separate the functions of stretching the wires and establishing their planarity. The grid frame is under tension and holds the wires, and is allowed to deform. A cylindrical flattener with an optically flat end surface (figure 7) is then brought into contact with the stretched wire surface by turning three \#0-80 screws until all the wires just deflect. This deflects all the wires into planarity with only a tiny stress on the flattener, so its accuracy is unaffected.

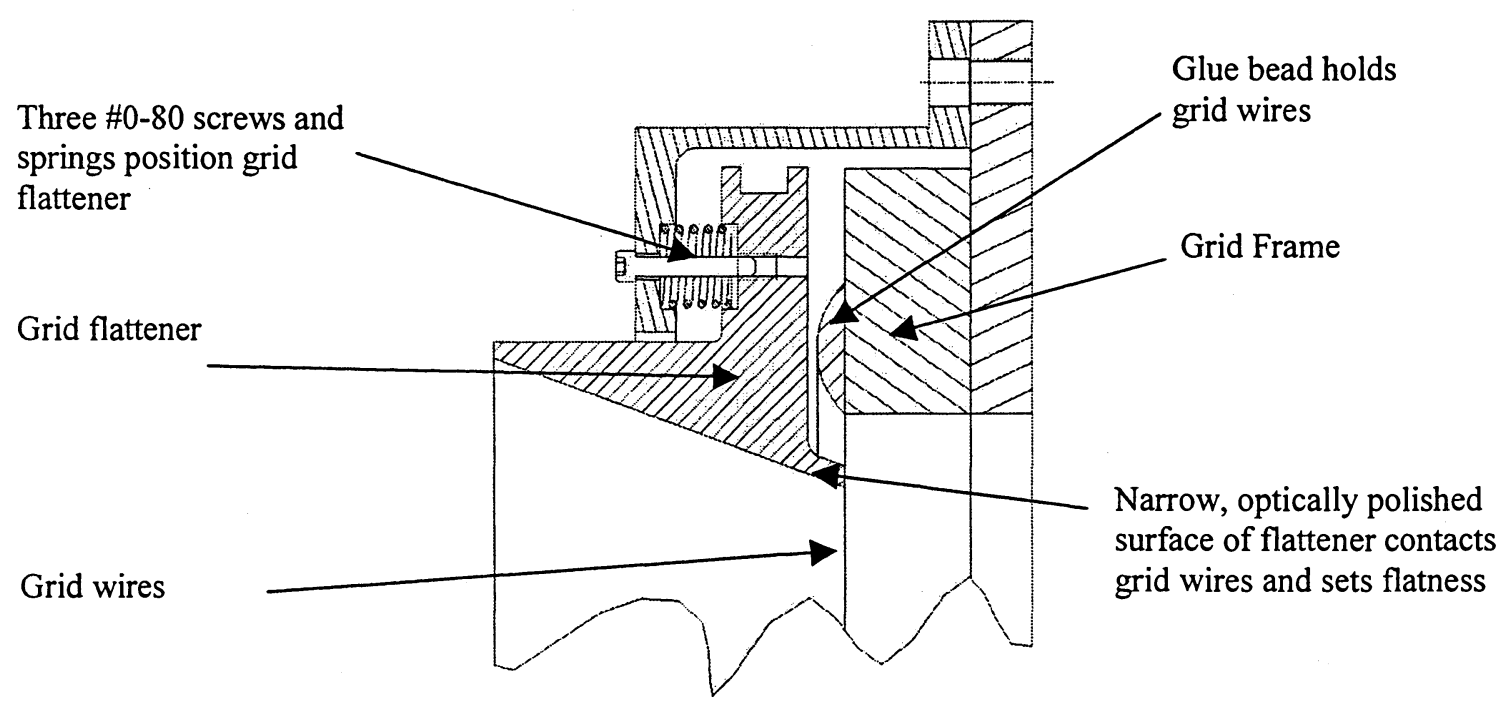

Figure 7) : Detail of grid assembly showing the grid flattener.

Examination under a measuring microscope revealed that the flattener improved the planarity of a commercial polarizing grid (reference 7) from a local peak-to-peak roughness of XX $\mu \mathrm{m}$ and overall deviation from an ideal plane of XX $\mu \mathrm{m}$ to $2 \mu \mathrm{m}$ flatness.

\section{CONCLUSION}

The kinematic, flexure-based linear stage described here worked well for establishing precise, linear motion during room-temperature testing as part of the Hertz VPM instrument. For cryogenic applications, this technology has the following potential advantages over existing linear motion devices: 1) It is entirely flexurebased: it uses no lubricants or rolling / sliding parts 2) All alignments can be done warm, 3) only one actuator / 
sensor pair is required, and 4) the control bandwidth is high and position noise is low because there is no crosscoupling between any controlled axes.

Additionally, the grid flattener described here is a very simple device that greatly improves the flatness of the wire polarizing grids.

\section{REFERENCES}

1) Gravity Wave ref

2) chuss polarization ref

3) VPM functioning ref

4) SPIFI ref

5) Piezo actuator ref DSM

6) cap sensor ref

7) militek polarizing grid reference 